\title{
Qué drogas se prefieren para las relaciones sexuales en contextos recreativos
}

\author{
Amador Calafat*; Monserrat Juan*; Elisardo Becoña*, **; Alejandro Mantecón*** \\ * Socidrogalcohol \\ * Universidad de Santiago de Compostela \\ *** Universidad de Alicante \\ Enviar correspondencia a: \\ Amador Calafat. Socidrogalcohol. Rambla, 15, 2 , 3ª 07003 Palma de Mallorca (España). Correo e.: adicciones@socidrogalcohol.org
}

Recibido: Noviembre de 2006 Aceptado: Octubre de 2007

\section{RESUMEN}

Entre los adolescentes el consumo de alcohol y otras drogas está frecuentemente vinculado al contexto recreativo nocturno, al igual que la búsqueda y experimentación en sexualidad. Cada una de las sustancias tiene efectos en el comportamiento, en la experiencia sexual y en las consecuencias. Aquí se explora cómo experimentan los adolescentes españoles la relación y el rol que ocupan las drogas en la experimentación de la sexualidad en estos contextos recreativos.

Se ha utilizado metodología cualitativa para explorar la relación entre sexualidad y drogas en 100 jóvenes de ambos sexos de 4 ciudades españolas, que les gustase salir los fines de semana y con experiencia sexual. Se les localiza mediante el sistema de bola de nieve y se les hace entrevistas en profundidad. Los resultados confirman la importancia que adquiere el contexto recreativo nocturno en la búsqueda y experiencia sexual entre los jóvenes. Los jóvenes tienen una idea muy precisa de cómo cada una de ellas funciona en relación con distintas prácticas sexuales. El alcohol es con mucho la más popular y la que tiene más ventajas en las cuatro prácticas sexuales analizadas (facilitar el encuentro sexual, involucrarse en experiencias más arriesgadas, aumentar la excitación y prolongar la relación sexual), excepto para prolongar la relación que se prefiere la cocaína. El cannabis no gusta porque relaja y no hay interés en el éxtasis por sus efectos sobre la sexualidad. A las mujeres les gusta mucho el alcohol para experiencias arriesgadas, aumentar sensaciones y prolongar sexo y utilizarían menos cocaína.

Palabras clave: sexo, drogas recreativas, metodología cualitativa, efectos drogas.

\section{ABSTRACT}

Many people associate the use of alcohol and other drugs with sexuality. It is common to find that each drug is associated with a specific effect on sexuality. Weekend recreational nightlife settings are increasingly important places for the young, and frequented by them more and more in search of sex and drug-taking opportunities. In this research we are interested in the role the young attribute to recreational drugs with regard to their sexual practices. We interviewed a sample of 100 young people from four Spanish cities, using a questionnaire with both open and closed questions. Snowball sampling was used to find those who had had sexual experience, who had taken recreational drugs and who liked going to discos, bars, etc. at the weekend. We found that these young people have a very precise idea of how each drug functions within sexuality. Considering all four parameters analysed, alcohol is by far the most popular (to initiate the sexual encounter, for more unusual or the "hottest" experiences, to increase arousal, and to prolong sex), though in the last case in particular the preferred drug was cocaine. Cannabis does not interest them because of its relaxing effects, while ecstasy is chosen more for remaining active and enjoying oneself than for its sexual effects. Women use alcohol more than men (mainly to increase arousal, when they want unusual sex or to prolong sex) and use cocaine less.

Key words: sexual practices, recreational drugs, drug effects, qualitative methodology.

\section{INTRODUCCIÓN}

$\mathbf{L}$ a adolescencia es un período de iniciación y esto incluye muchas veces la iniciación en el sexo y las drogas. La mayoría de los jóvenes españo- les empiezan a tener relaciones sexuales entre los 16 y los 20 años (INE, 2004; Espinosa y Ochaita, 2003). Estudios recientes demuestran que en la última década se ha duplicado el porcentaje de jóvenes de entre 15 y 17 años con experiencias sexuales, tanto en 
chicas como en chicos (INJUVE, 2005). Además, la primera relación sexual no se convierte en un acontecimiento aislado, ya que el $90 \%$ de los jóvenes que ya han tenido una primera experiencia sexual afirman haber mantenido otras experiencias en los últimos 12 meses (INJUVE, 2004). Este incremento de la frecuencia de las relaciones sexuales debe situarse en el marco de los importantes cambios sociales, culturales y políticos que han tenido lugar en las sociedades occidentales desde el final de la segunda guerra mundial. Se puede destacar su progresiva disociación de las normas morales tradicionales y, al mismo tiempo, su mayor vinculación con un sistema de motivaciones que valora la sexualidad como una experiencia placentera y prestigiosa que depende cada vez más de decisiones estrictamente individuales (Giddens, 1995; Bantman, 2000).

La más intensa vida sexual de los adolescentes, desde un punto de vista de salud pública, tiene relación con algunos problemas. Por un lado están las prácticas sexuales de riesgo. El contacto sexual aparece en varios países de la Europa occidental, entre ellos España, como una vía de nuevas infecciones de VIH (UNAIDS, 2006). Estudios empíricos demuestran cómo se incrementaron en Europa los diagnósticos de VIH y de otras enfermedades de transmisión sexual entre 1995 y 2000 (Nicoll y Hamers, 2002). El índice de interrupciones voluntarias de embarazos (motivados por conductas sexuales de riesgo) entre las españolas menores de 19 años también ha crecido, pasando en 2005 a una tasa de 10,9, el doble de la que se registraba en 1997 (MSC, 2005). Por otro lado algunos autores señalan la existencia de una relación entre este tipo de problemas y el consumo de drogas (Donovan y McEwan, 1995; Hibell, Andersson, Bjarnasson, Ahlstrom, Balakireva, Kokkevi et al, 2004), si bien, estos vínculos no son siempre fáciles de demostrar. Por ejemplo, en el caso del alcohol existen investigaciones contradictorias, lo cual seguramente está relacionado con que hay bastantes variables que pueden afectar a los resultados: el tipo de muestra seleccionada, la orientación sexual, la edad, las campañas preventivas, etc. Leigh (2002) ilustra esta complejidad descubriendo una correlación significativa entre la ingesta de alcohol y la no adopción de medidas protectoras durante la primera relación sexual, sin embargo esta asociación deja de ser significativa cuando se estudian las relaciones posteriores.

Tanto la expansión de la vida sexual como del consumo de drogas no son ajenos a la expansión de otro fenómeno social importantísimo en el mundo occidental que es el modelo recreativo de las noches de los fines de semana, y que algunos han denominado modelo de diversión hegemónico (MDH) (Calafat, Fernandez, Juan, Anttila, Bellis, Bohrn et al, 2004). Este $\mathrm{MDH}$ se ha propagado de forma acelerada en Europa desde mediados de los 80, a partir en gran medida de los modelos de diversión nocturna creados en los enclaves turísticos mediterráneos. Todavía hoy los centros turísticos ejercen una considerable influencia y, de hecho, los jóvenes que viajan a lugares turísticos aumentan durante la estancia la frecuencia de sus relaciones sexuales, el número de parejas con que se relacionan y, también, las prácticas sexuales de riesgo y el consumo de drogas (Collin, 1997; Elliott, Morrison, Ditton, Farral, Short, Cowan et al, 1998; Bellis, Hale, Bennet, Chaves y Kilfoyle 2000; Bellis y Huges, 2004; Bellis, Hughes, Dillon, Copeland y Gates, 2007). En un estudio realizado durante varios años a jóvenes británicos que han viajado a Ibiza se demuestra que la motivación principal para realizar este viaje es la participación en actividades recreativas nocturnas (sobre todo en discos, pubs...), lo cual se traducirá en la práctica en un mayor número de relaciones sexuales, con más parejas distintas y mayores consumos de alcohol y drogas (Bellis et al, 2000).

Esta forma de diversión es muy popular desde hace años entre los jóvenes españoles (Calafat, Juan, Becoña, Fernández, Gil, Palmer et al, 2000; Rodríguez, Agulló y Agulló, 2003; Comas 2005; López, Cachón, Comas, Andreu, Aguinaga y Navarrete, 2005) y recibe coloquialmente el nombre de salir de marcha. Según la Encuesta Escolar española 2004 (PNSD 2004), realizada a partir de una muestra representativa de jóvenes escolarizados de 14 a 18 años, más de la mitad ha "salido de marcha" durante el último mes. En su última salida un 44,8\% se emborrachó, un $26,4 \%$ fumó cannabis, un 3,8\% consumió cocaína, un 1,4\% usó éxtasis y otro 1,4\% alucinógenos. El horario de regreso a casa está relacionado con el consumo de sustancias. Refiriéndonos únicamente a las embriagueces, casi la mitad (un 47,2\%) de los que se emborracharon regresaron a su casa después de las 4 de la madrugada, mientras que tan sólo un 17,9\% corresponde a los que regresaron antes de las dos. Otros estudios también avalan que el consumo de alcohol y otras drogas entre quienes participan en este tipo de actividad recreativa es mayor que el corresponde al conjunto de la población (EMCDDA 2002, 2006).

Los adolescentes y los jóvenes interpretan el contexto recreativo como un espacio al cual se acude con expectativas de ligar o de lograr un encuentro sexual (Megías, 2003; Megías, Rodríguez, Méndez y Pallares, 2005). En un estudio con 2.550 entrevistados en contextos recreativos de 9 ciudades europeas de distintos países, se observa que el objetivo de 'buscar sexo' es una motivación importante para salir a divertirse los fines de semana para el 27,8\%, buscar pareja para el 28,9\% y consumir drogas para el 18,7\% (Calafat, Bohrn, Juan, Kokkevi, Maalsté, Mendes et al, 1999). En el contexto específico de los ambientes recreativos hay estudios que muestran que cuando hay perspectiva de encuentros sexuales ello puede derivar hacia un mayor consumo de drogas (Elliott et 
al, 1998; Brook, Brook, Pahl y Montoya, 2002; Guisado, Vaz, Fernández-Gil, Pacheco, Graell y López-lbor, 2000; Parsons, Kelly y Wells, 2006; Rodríguez, Hernán, Cabrera, Romo, García y Gutiérrez, 2006). Son muchos los jóvenes que valoran las sustancias (muy en especial el alcohol, pero también otras como cannabis, cocaína o éxtasis) por sus efectos facilitadores de logros sexuales (Abrahamson, 2004; Sumnall, Cole y Jerome, 2006) que abarcan un amplio abanico de objetivos que van desde la relajación hasta una completa desinhibición.

Existe por tanto un cierto conocimiento sobre la relación entre sexo y drogas. Pero esta relación está menos estudiada refiriéndola directamente a los ambientes recreativos típicos de las noches de fin de semana. Se explorará en concreto las relaciones que establecen los jóvenes en estos contextos entre las drogas más habituales y la consecución de determinadas metas sexuales como pueden ser facilitar los encuentros, aumentar las sensaciones, prolongar las relaciones e involucrarse en relaciones sexuales menos usuales.

\section{MÉTODO}

\section{Muestra}

Los entrevistados se seleccionaron utilizando el sistema de bola de nieve (Kemmesles, 2000). En el campo de los estudios sobre consumo de drogas este tipo de muestreo no probabilístico ha resultado muy útil debido a las dificultades que plantea identificar a los entrevistados y localizarlos en ambientes diferentes a los de consumo habitual que, muchas veces, se hallan ocultos (Coleman y Cater, 2005; Kemmesles, 2000; Neale, Allen y Coombes, 2005). La práctica de la bola de nieve consiste en la identificación de unos informantes iniciales que, a su vez, nos ponen en contacto con otros miembros de la población objetivo a los que nos dan la posibilidad de acceder (Goodman, 1961). La gran ventaja de esta metodología es que permite el acceso comprensivo a una determinada esfera de la realidad social captando el significado que adoptan los propios sujetos que en ella se hallan inmersos (Neale et al, 2005). Este procedimiento cualitativo tiene enormes ventajas cuando se utiliza adecuadamente, aunque también tiene sus limitaciones.

El criterio que utilizamos para dar por finalizada la muestra fue el de la saturación de la información recogida, es decir, cuando la información que nos proporcionaban los nuevos entrevistados se volvía redundante y ya no aparecía nueva información.

El total de la muestra está formada por 100 personas. Todas reúnen los tres criterios de selección: a) tener 20 años como máximo, b) haber tenido alguna experiencia sexual, y c) ser consumidores de alguna droga legal o ilegal. La muestra final está formada por 43 varones y 57 mujeres. Las personas tienen edades comprendidas entre los 15 y los 20 años, de ellos 54 tienen 18 años o menos y 46 tienen 19 y 20 años. El trabajo de campo se realizó entre enero y mayo de 2006. Para mejorar la representatividad de la muestra se seleccionaron informantes de cuatro ciudades españolas (Madrid, Palma de Mallorca, Valencia y Santiago de Compostela). En cada una de estas ciudades se contactaron inicialmente cuatro adolescentes en locales recreativos nocturnos, de los cuales dos debían ser varones y dos mujeres. Uno de los varones y una de las mujeres se contactaron en un local recreativo muy popular (mainstream) entre los adolescentes (discotecas, salas de fiesta, galas de tarde), y el otro varón y la otra mujer se contactaron en locales más visitados por jóvenes adscritos a alguna subcultura (han sido básicamente bares musicales y pubs, donde predominan la música reggae, heavy, ghotic, tecno).

\section{Entrevistas}

La técnica de recogida de datos utilizada fue la entrevista en profundidad. Se utilizó un guión semiestructurado, con preguntas abiertas dirigidas a conocer el significado que dan los entrevistados acerca de la influencia que ejerce el consumo de diferentes drogas en el proceso de un encuentro sexual. Para preparar mejor el guión de la entrevista, formar al equipo de entrevistadores y crear los criterios de la muestra y del contexto en el que se produciría el encuentro con los entrevistados, se llevó a cabo una aproximación previa mediante la realización de cuatro grupos de discusión integrados por adolescentes que "salen de marcha" frecuentemente.

Todos los entrevistados aceptaron participar en el estudio de forma voluntaria, después de que se les garantizara preservar su anonimato. Los participantes tenían el derecho a no contestar a las cuestiones planteadas y a interrumpir o dar por finalizada la entrevista cuando quisieran. Todos nos dieron su consentimiento para grabar la entrevista en cinta magnetofónica. Las grabaciones se transcribieron en su totalidad y se analizaron con la asistencia técnica del programa informático Atlas.ti 4.2. Las entrevistas duraron entre dos y cuatro horas, dependiendo del tiempo requerido por cada entrevistado para exponer sus argumentos ante las cuestiones que les proponíamos.

Después de transcribir la información recogida, se realizó una operación analítica y otra teórica. La primera ha consistido en la codificación de los textos, a partir de la generación de códigos temáticos y de su agrupación en familias de códigos. A continuación se 
han seccionado discursos producidos en torno al alcohol y a cada una de las otras drogas, para, posteriormente, reclasificar el material reunido asociando ideas y descubrir tipologías, posicionamientos y líneas argumentales, siguiendo a Sandelowski (1995). El análisis de la información recopilada se ordenó en torno a la percepción que los jóvenes pertenecientes a la población objetivo tienen de cada una de las drogas de consumo más frecuente $y$, específicamente, de su influencia en las relaciones sexuales. Ordenadas de mayor a menor consumo son: el alcohol, el cannabis, la cocaína, el éxtasis y un grupo muy minoritario formado por anfetaminas y alucinógenos (PNSD, 2004). La operación teórica ha consistido en la elaboración de un argumento explicativo en el que se sintetice el esfuerzo interpretativo, lo que se intenta en las próximas líneas. Las limitaciones de espacio de este artículo nos obligan a escoger una muestra muy reducida y selectiva de fragmentos ejemplares de las entrevistas, seleccionando aquellos que realmente expresan el sentido general de las líneas discursivas identificadas en el conjunto de las entrevistas.

\section{RESULTADOS}

\section{¿Cómo ven los adolescentes los vínculos entre el sexo y las drogas?}

Aunque la mayoría de los entrevistados reconoce ligar con frecuencia, no todos entienden lo mismo por 'ligar'. Para algunos -sobre todo para los varones- ligar significa directamente tener una relación sexual. Pero para otros es más bien un inicio de algo, un flirteo, un nuevo contacto que puede o no ir a más.

\section{Ligar es:}

"Mojar, liarse, tirarse a alguien, Liarte con alguien, triunfar, pillar cacho, Flechazo o polvo rápido."

"Es un juego, miradas...no sé, que haya un feeling. Aunque no vaya a culminar en nada, ligar no tiene porque ser echar un polvo."

“...una complicidad bastante grande, ponerte a hablar, bailar con ella, no es necesario llegar a enrollarte. Simplemente con bailar muy pegado."

"Gustarle a un chico mientras le estás bailando con movimientos obscenos."

En las entrevistas se admite que la mejor forma de ligar es ir directamente a ciertos locales en los que, previamente, se sabe que es habitual que quienes acuden estén bajo la influencia del alcohol o las drogas. Algunos entrevistados mencionan que la sexualidad que se da en esos contextos es muy del momento, se busca el placer inmediato y sin vínculos emocionales. Utilizan la expresión del: 'aquí te pillo y aquí te mato'. Con ello definen un modelo de sexualidad 'instantánea' del momento, que tiene lugar en el propio contexto o en las cercanías, como parte del ritmo nocturno. Todos los entrevistados confirman la importancia que adquiere el contexto recreativo nocturno como espacio para ligar y acceder a relaciones sexuales:

\begin{abstract}
"Cuando busco sexo voy a un sitio de niñas pastilleras, porqué hay sexo fácil. Son las típicas niñitas que les invitas a una raya y te las tiras."

"En las discotecas es fácil porque la gente va muy borracha o drogada y siempre es más receptiva y menos quisquillosa."

"Hay dos sitios buenos para conseguir sexo. Donde están las zorras pijas, en los que está de moda el éxtasis y el rollito de todos somos amigos. Aunque vete a saber lo que te encuentras. Y luego cualquier sitio en donde haya fiesta universitaria. Son el circo donde los animales se emparejan."
\end{abstract}

Para todos los entrevistados la relación entre sexualidad y drogas es estrecha y evidente. En el contexto en el que quedan insertos los vínculos que unen sexualidad y diversión cada sustancia adquiere una función específica. Esas funciones son el resultado de las propias experiencias de los propios jóvenes o de sus amigos, y de los efectos que les atribuyen a cada sustancia. Hay preferencias hacia algunas sustancias y críticas hacia otras. En todo caso, lo que no hay es indiferencia por ninguna. Lo que más llama la atención es que las distintas drogas son valoradas como "buenas" o "malas" en función de si logran el objetivo de mejorar en alguna dimensión las relaciones sexuales, pero no hay referencias a valores morales o éticos sobre su consumo. Apenas se pueden identificar críticas sobre el hecho de que se utilicen drogas para fines sexuales. Una excepción es la siguiente:

\footnotetext{
"Pues todas las drogas llevan a una forma artificial de establecer contacto con otra persona y no hay mérito propio en el acto sexual, y si disfrutas es por las drogas, no hay un verdadero y sano placer. No sé, tampoco es que haya probado muchas pero ni el éxtasis es demasiado sobado y ni los alucinógenos, no me mola follar con un duende mirándome."
}

En su gran mayoría los comentarios de los adolescentes en relación a las drogas son descriptivos de sus efectos, o bien, acerca de cómo las distintas sustancias facilitan o dificultan el logro de los objetivos propuestos al consumirlas. En nuestro caso se trata de cómo ayudan a conseguir una relación sexual con 
alguien y de cómo influyen en la duración, la excitación y en el orgasmo. Estos comentarios se enmarcan en la lógica de lo que tradicionalmente se ha interpretado como la dimensión masculina de la sexualidad, a la que parece que se han apuntado también las mujeres. Algunas opiniones al respecto son:

"Los hombres con esas sustancias se abren más, se atreven a iniciar una conversación con nosotras, pero también la cagan más porque así controlan menos."

\begin{abstract}
"Hay personas que lo que buscan es probar cosas nuevas y si aún encima te gusta un chico que tiene drogas, pues te lías con él y así es más fácil conseguir esas drogas."

"Invitar a algo te da como un vínculo, es un acercamiento. Por ejemplo, si tienes marihuana, te es más fácil acercarte, y compartir y fumar y eso une bastante, estás más suelto y facilita mucho las cosas."

"Lo peor es el alcohol en exceso y los porros, porque no se te levanta, no te enteras, no te corres. El porro porque me atonta y el speed porque me pone borde."

"He probado el cannabis, pero no sé, no te podría hablar mucho. En el caso del alcohol mejora la duración, aguantas más tiempo sin eyacular, aunque también puede ser el caso contrario y empeorarla. Por lo que me han contado la cocaína es la hostia, incluso creo que hay parejas que necesitan estar puestos de cocaína para tener relaciones."
\end{abstract}

\section{Valoraciones de cada droga según sus efectos sobre la sexualidad}

Se recogen aquí las razones por las que nuestra población elige determinadas drogas en función de conseguir determinados logros en sus prácticas sexuales. Se exploraron concretamente cuatro aspectos de las relaciones sexuales: facilitar su inicio; el deseo de prácticas sexuales menos usuales o más arriesgadas; el aumento de la excitación; y la prolongación de las sensaciones.

El alcohol no solo es la droga más consumida, es también la que interviene más habitualmente en la sexualidad. El alcohol es la sustancia más valorada por los jóvenes de nuestra muestra en relación con el sexo, excepto a la hora de prolongar la relación, aspecto para el que la cocaína es la sustancia preferida. El alcohol es la sustancia más utilizada para iniciar las relaciones, para los primeros contactos. También es una estrategia para lograr sexo, ya que los varones creen que baja la resistencia de algunas chicas que se muestran tímidas o indecisas. Del mismo modo, algu- nas de las chicas buscan por sí mismas esos efectos a través del alcohol: la sensación de ser atrevidas y de eliminar la timidez.
"Sé de muchos hombres que creen que si una chica está borracha va a ser más fácil que se enrolle con ellos."

"Del alcohol me gusta la sensación de ir borracha, de no acordarme de nada y pasármelo bien."

"El alcohol es la mejor, porque hace que te desmadres. Porque te pone más cachonda, con más ganas. El alcohol facilita el acercamiento. Mejora las relaciones sexuales porque te da más facilidad para soltarte."

En todo caso, llama la atención como las mujeres entrevistadas hacen mucho más uso del alcohol que los hombres para gestionar su sexualidad. Según explican, esto es así tanto para facilitar el contacto como para tener prácticas sexuales más arriesgadas, buscar una mayor excitación o prolongar la relación sexual.

\footnotetext{
"Yo creo que la mejor es el alcohol. Mejora las relaciones sexuales porque te da más facilidad para soltarte. Porque hace que te desmadres."

"El alcohol me gusta porque estás más desinhibida y te quita la vergüenza."

"El alcohol me va. Al estar pedo ya no tienes vergüenza a nada, te da igual hacer lo que haya que hacer."
}

También hemos podido identificar un discurso de las mujeres que critica el uso del alcohol por parte de los hombres como medio para facilitar los encuentros sexuales. Muchos varones beben porque desinhibe y creen que así van a conseguir más fácilmente alguna relación. Sin embargo, algunas mujeres reconocen alejarse de hombres excesivamente embriagados y difícilmente los ven como posibles parejas sexuales. Es decir, hay varones que beben para desinhibirse pero si se pasan y se embriagan ello puede transformarse en un problema para conseguir ligar. El resultado es que los hombres se pueden encontrar con problemas para lograr una erección a la hora de abordar la relación. Entonces, para los varones, el alcohol puede convertirse en un arma de doble filo.

En cambio, el discurso masculino es opuesto al de las mujeres. Para los varones el que una chica esté embriagada es visto muchas veces como una ventaja de cara a conseguir tener relaciones sexuales con ella. La estrategia más clásica para crear un contacto que puede dar lugar a una relación sexual es invitar a una copa. La estrategia también funciona entre adolescentes, muchos saben que las chicas cuando beben 'se sueltan'. Algunos de los siguientes comentarios reflejan lo mencionado: 
"Es mejor no consumir alcohol porque en mi caso el alcohol puede subirme la libido pero luego no respondo en la relación sexual, y no disfrutas por el estado en el que está el cuerpo. Y con el éxtasis me pasa lo mismo."

"Yo creo que ninguna droga mejora las relaciones sexuales, al contrario, las empeoran. Por ejemplo, si estás borracho te mareas y lo único que te apetece es dormir."

"Los hombres no pueden consumir mucho alcohol porque no levantan. La cocaína va mejor porque te da subidón. Está más despierto al principio, pero en el momento más íntimo no es tan buena porque el chico se va."

"A mí que te inviten no me gusta. Yo tengo una amiga que un tío le invitó a más de una copa y luego ella se sintió obligada a irse con él."

El cannabis es una droga que crea opiniones contrapuestas entre los jóvenes que participaron en el estudio. Pocos lo valoran como aliado sexual, pero quienes sí lo hacen argumentan que aumenta la excitación, hace estar más sensible y receptivo. Las mujeres suelen ser quienes más hacen esta valoración positiva del cannabis en relación al sexo, pues, tal y como explican, produce una relación más sensitiva que activa. La mayoría de los entrevistados consideran que el cannabis adormece, atonta, y esos efectos son vistos como contrarios a la relación sexual que muchos pretenden en estos contextos recreativos.

"A mí me va la marihuana y un poco de alcohol, porque me excitan más. Y en chicos yo creo que... no sé."

"Los petas hacen que el sexo sea una experiencia fantástica."

Luego se observan algunos comentarios críticos sobre el consumo de cannabis. Básicamente se centran en que induce a la inactividad, pasividad, relajación, etc. y, por ello, actúan de forma contraria a lo que algunos pretenden en una relación sexual. Algunos comentarios son:

"Con un porro te puede dar un gatillazo y aparte estás como atontado".

"Dicen que con los porros va peor. Debe ser verdad porque la gente cuando va fumada se queda tonta."

"Es mejor no consumir porros porque te adormilan, aunque también depende de la persona."

La cocaína tiene una larga historia como droga con supuestas ventajas para la actividad sexual, por ejemplo, por su supuesto efecto afrodisíaco (Gay, New- meyer, Perry, Johnson y Kurland, 1982). Hay quienes creen que la cocaína ayuda a retrasar el orgasmo y prolongar la actividad sexual (Buffum, Moser y Smith, 1988). En nuestro estudio vemos como la cocaína es valorada en segundo lugar, detrás del alcohol, y en cierto modo, como complemento de éste. La mayoría piensa que facilita el inicio de las relaciones (atreverse, desinhibirse...), y una cuarta parte de los entrevistados consideran que favorece el acceso a prácticas sexuales más atrevidas y que aumenta la excitación y las sensaciones. De hecho, varios han especificado lo excitante que es lamer cocaína directamente del glande. La cocaína es valorada muy positivamente, sobre todo por y para las mujeres.

"Y la cocaína, aunque hay gente que dice que al hombre hace que le cueste más que se le levante. Pero para la mujer creo la cocaína es mejor porque hace que se alargue más la relación sexual."

Al ser una droga que da prestigio y poder (Calafat, Juan, Fernández, Gil y Llopis, 2001) ello facilita que sea utilizada por los varones como anzuelo para atraer posibles parejas sexuales. De forma más o menos explícita se produce una especie de intercambio entre dar cocaína a cambio de sexo. Este intercambio también ha sido mencionado al referirse los entrevistados a otras sustancias, pero de una manera menos evidente que con el caso de la cocaína. Otras cuestiones que se mencionan es que desinhibe, hace ser más atrevido y prolonga la relación sexual. Algunas mujeres destacan especialmente estos efectos positivos, aunque la prolongación de la relación es a veces vista como un inconveniente, pues acaba siendo incomodo y hasta doloroso.

Algunos comentarios de los entrevistados expresan los efectos positivos que valoran en la cocaína:

"A lo mejor explícitamente lo de si te doy drogas te vienes conmigo, no funciona tanto. Pero lo de si coqueteo el otro me invita a drogas, sí que es muy normal."

"Yo lo he hecho, no lo dices pero si tienes coca la tía se va contigo."

"Conozco la cocaína. En los chicos no lo sé. Pero en mi caso, sí pongo más pasión."

"Yo... lo he hecho con una tía que iba puesta de coca y ellas se ponen mucho más."

"La cocaína a veces va muy bien y otras nada. Para las tías sí. Por la energía."

"La cocaína porque se aguanta más. Popper y cocaína son increíbles, con la coca mas potencia, con el popper más sensaciones." 
"Alcohol y cocaína; el alcohol te quita la vergüenza y las tías son más fáciles, la coca te pone más fuerte, te da mas aguante."

Pero también hemos identificado comentarios críticos que surgen a propósito de experiencias personales o de allegados. Al respecto, los comentarios más repetidos giran entorno al efecto negativo que causa la cocaína a la hora de lograr la erección y el orgasmo masculino. Tanto los hombres como algunas de las mujeres valoran esos efectos de manera crítica.

"Con la cocaína dicen que ni se te levanta. Pero las mujeres se desinhiben más. Al estar superborrachas se enrollan con gente con quien no lo harían de estar normal."

"La cocaína no es buena para mantener relaciones sexuales porque es para ir más lento".

El éxtasis, en contra de lo esperable, es de las sustancias menos seleccionadas por sus efectos positivos sobre la sexualidad, a pesar de que ha sido etiquetada como "droga del amor" por algunos de sus supuestos efectos (Zemishlany, Aizenberg, Weizman, 2001). Son pocos los que mencionan que con el éxtasis mejoran las relaciones sexuales, e incluso existen referencias negativas. Los comentarios siguientes sirven como resumen de la valoración más extendida sobre esta sustancia.

"Yo creo que aunque no sientas lo mismo, las pastillas van bien, pero no porque te apetezca más o te modifiquen algo sino porque tienes más ganas de estar con alguien."

"Quizás al principio de la relación con alguien el éxtasis va bien, pero a la hora de las relaciones sexuales es un desastre."

"Las pastillas me van mal porque te ponen medio loco."

En la tabla 1 se sistematizan los resultados obtenidos.

Tabla 1. Percepción de la influencia de las drogas en las relaciones sexuales.

\begin{tabular}{|c|c|c|c|c|}
\hline & Alcohol & Cocaína & Cannabis & Éxtasis \\
\hline Facilitar el inicio & $\begin{array}{l}\text { Sustancia más valorada. } \\
\text { Mujeres y hombres } \\
\text { valoran positivamente } \\
\text { su efecto desinhibidor, } \\
\text { especialmente sobre las } \\
\text { mujeres. } \\
\text { Las mujeres advierten } \\
\text { de que puede convertir- } \\
\text { se en un arma de doble } \\
\text { filo para los hombres. }\end{array}$ & $\begin{array}{l}\text { Complemento al alcohol. Valo- } \\
\text { rada positivamente. } \\
\text { Se asocia a posiciones de sta- } \\
\text { tus y privilegio, lo que ayuda al } \\
\text { cumplimiento de una función } \\
\text { clave como mecanismo implí- } \\
\text { cito de intercambio: cocaína a } \\
\text { cambio de sexo. }\end{array}$ & $\begin{array}{l}\text { Reduce las habilidades } \\
\text { para la interacción, } \\
\text { produce pasividad. }\end{array}$ & Críticas. \\
\hline $\begin{array}{l}\text { Prácticas menos } \\
\text { frecuentes }\end{array}$ & Sustancia más valorada. & $\begin{array}{l}\text { Valorada positivamente. } \\
\text { Complemento al alcohol. }\end{array}$ & $\begin{array}{l}\text { Críticas: adormece, } \\
\text { atonta, produce pasi- } \\
\text { vidad. }\end{array}$ & Críticas. \\
\hline $\begin{array}{l}\text { Aumento de la } \\
\text { excitación }\end{array}$ & Sustancia más valorada. & $\begin{array}{l}\text { Valorada positivamente. Espe- } \\
\text { cialmente es señalado su } \\
\text { efecto positivo por y para las } \\
\text { mujeres. } \\
\text { Crítica: puede dificultar la } \\
\text { erección y el orgasmo mas- } \\
\text { culino. }\end{array}$ & $\begin{array}{l}\text { Las mujeres, princi- } \\
\text { palmente, señalan su } \\
\text { efecto positivo. }\end{array}$ & Críticas. \\
\hline $\begin{array}{l}\text { Prolongación de } \\
\text { la relación }\end{array}$ & $\begin{array}{l}\text { Valoración menos entu- } \\
\text { siasta. }\end{array}$ & $\begin{array}{l}\text { Sustancia más valorada. Aun- } \\
\text { que puede llegar a derivar en } \\
\text { problema al provocar dolor. }\end{array}$ & $\begin{array}{l}\text { Críticas: adormece, } \\
\text { atonta, produce pasi- } \\
\text { vidad. }\end{array}$ & Críticas. \\
\hline
\end{tabular}

Fuente: elaboración propia. 


\section{DISCUSIÓN}

El presente estudio trata de explorar y entender como los adolescentes españoles implicados en lo que hemos denominado el Modelo de Diversión Hegemónico perciben y utilizan la relación entre la sexualidad y las drogas. El contexto recreativo se ha ido convirtiendo en un espacio de socialización clave para los jóvenes actuales, donde se elaboran los significados y los marcos culturales que orientan los estilos de vida (Sutherland y Shepherd, 2002). Los estudios culturales muestran como los consumos y las expectativas de los jóvenes hacia las distintas sustancias quedan integradas en una dinámica muy compleja donde intervienen elementos del ambiente, las emociones y las conductas (Gilbert y Pearson, 2003; Green, 1997).

Los jóvenes de nuestro estudio se sienten cómodos al hablar del tema y casi todos piensan que el consumo de algunas drogas facilita o interfiere de alguna manera la actividad sexual. Son capaces de expresar su punto de vista sobre cada una de las drogas y sobre aspectos concretos de la sexualidad que ellos consideran que mejoran o empeoran. Tanto las drogas como el sexo son vistos casi exclusivamente como instrumentos a través de los cuales pasarlo bien, lograr diversión y placer. Hasta qué punto esta visión instrumental, positiva y poco crítica viene reforzada por ser jóvenes que frecuentan contextos recreativos es difícil de saber.

El alcohol es la sustancia preferida para casi todos los supuestos planteados. Es frecuente su uso para facilitar las relaciones - recordemos que la estrategia más clásica para crear un contacto que puede dar lugar a una relación sexual es invitar a una copa (Traeen, Hovland y Odegard, 1998)-, disminuir las inhibiciones, para tener relaciones sexuales más arriesgadas $\mathrm{y}$, en menor medida, como prolongador de la relación sexual. Diversos estudios han destacado la importancia de las expectativas individuales sobre los efectos del alcohol en el comportamiento sexual (Leigh, 1990; Friedman, McCarthy, Förster y Densier, 2005) y los efectos diferenciales en función de distintos grupos sociales y situaciones contextuales. De hecho, en un estudio dos de cada tres relaciones examinadas el uso de alcohol se asociaba con la toma de más riesgos entre los consumidores que esperaban que el alcohol les aumentaría las relaciones sexuales de riesgo (Dermen, Cooper y Agocha, 1998). Los hombres y mujeres, debido a las diferentes expectativas sociales que existen sobre ellos pueden presentar distintas conductas incluso dentro de un mismo contexto social (Wilsnack y Wilsnack, 2002). En nuestro estudio se ha revelado muy destacable la posición privilegiada que las mujeres conceden al alcohol y, en concreto, por su supuesto efecto afrodisíaco. Esto es interesante pues, aunque sí es tenida en cuenta como una sustancia que facilita las relaciones sexuales y está presente en muchos de los rituales sociales en los que se inicia una relación, se cita pocas veces como una sustancia propiamente afrodisíaca (Traeen et al, 1998).

La cocaína ocupa el segundo lugar en las preferencias por sus efectos sobre la sexualidad. Los hallazgos coinciden con lo que ya se conoce como sustancia que aumenta la excitación y que prolonga -a veces en exceso- la relación sexual. La cocaína es valorada frecuentemente como un afrodisíaco (Buffum et al., 1988; Gay et al., 1982). Es reseñable el hecho de que las mujeres manifiestan especial predilección por esta sustancia y, especialmente, el reconocimiento de un cierto intercambio implícito de sexo por drogas. El hecho de que haya más hombres que utilizan la cocaína para facilitar el inicio de las relaciones puede tener que ver con este sistema oculto de intercambio.

El cannabis está muy asociado a una parte muy significativa de los movimientos contraculturales de los años 60. Dicha cultura, además de presentar una visión muy positiva de dicha sustancia, también promocionaba una sexualidad más hedonista y libre. Evidentemente, todo ello influía en crear un nexo entre el cannabis y la sexualidad. Teniendo en cuenta todo esto, la marihuana era descrita como sexualmente evocadora y gratificante (Cohen, 1982, Dawley, Winstead, Baxter y Gay, 1979). Al principio de los 80 la marihuana se asociaba con un estilo de vida marcado por una actividad sexual precoz y frecuente (Abel, 1981). Más recientemente, algunos autores señalan la necesidad de diferenciar entre las funciones del cannabis antes y después de la relación sexual (Buffum et. al., y 1988; Poulin y Graham, 2001). A pesar de todo este mito cultural que relaciona cannabis con la sexualidad, no parece que a nuestra muestra de consumidores le guste la relajación favorecedora de las relaciones sexuales que algún estudio señala en relación con esta sustancia. Más bien al contrario, la relajación es el aspecto más criticado, sobre todo por los varones. Hay que tener en cuenta que estamos ante unos jóvenes cuyas relaciones se establecen en el contexto del "salir de marcha", lo cual requiere adoptar una actitud particularmente activa que, al parecer, no viene facilitada por el uso de cannabis.

Una de las sustancias que más se popularizó en los años 90 en los contextos recreativos de fin de semana fue el éxtasis (MDMA). Se le atribuían efectos como mejorar el contacto con la gente, la capacidad para bailar y escuchar la música y, además, el de aumentar la sensualidad y la sexualidad (Beck y Rosenbaum, 1994; Zemishlany et. al., 2001). En los 90 todavía no se conocían suficientemente algunos 
de los problemas ligados con el uso del MDMA, por lo que algunos profesionales, preocupados por el posible descrédito de la 'droga del amor' y de la cultura dance -en unos tiempos en los que se generaliza la preocupación por la transmisión sexual del VIH-, insistieron mucho en diferenciar entre sensualidad y sexualidad, en un intento de desvincular el éxtasis de la sexualidad. Para estas personas justamente la experiencia con el éxtasis lo que hacía era ir más allá de la necesidad física de sexo (Russel, 1991; Lyttle y Montagne, 1992; Henderson, 1993). Sin embargo, es destacable en este estudio como el éxtasis ha sido la sustancia menos valorada para ser utilizada en las relaciones sexuales. Podría pensarse que la etiqueta 'droga del amor' y todo el interés que despertó en los 80 y los 90 por su relación con la sexualidad fuesen exageradas y muy influidas por las circunstancias sociales específicas de aquel momento (relación del éxtasis con el movimiento rave, dance, etc.). No obstante, algunos estudios más recientes encuentran que una tercera parte de los que consumen esta sustancia lo hacen por sus efectos sexuales (Sumnall et. al., 2006). En otro estudio con 35 jóvenes que les gusta salir los fines de semana encuentran que el 90\% dice que el deseo y la satisfacción habían aumentado moderadamente o mucho, mientras que el orgasmo se retrasaba pero se percibía como más intenso, pero el gran problema es que la erección estaba dificultada en un $40 \%$ de los hombres (Zemishlany et al, 2001). En otro estudio (McElrath, 2005) con 88 usuarios de MDMA los resultados son contradictorios: para una parte sentían una mayor cercanía emocional, pero sin sentir deseos de penetración, mientras que otros (sobre todo los gay y las mujeres bisexuales) sentían que aumentaba su excitación sexual. En una revisión sobre 24 estudios en que se mencionan efectos subjetivos del éxtasis, sólo en 5 de ellos se menciona el aumento de la excitación y el aumento de la sensualidad (Baylen y Rosenberg, 2006). En cuanto al presente, cabe pensar como explicación al bajo interés que despierta el éxtasis, que las expectativas y la forma de uso sean distintas entre la población recreativa española. Podría ser que al ser las salidas nocturnas en España más largas y más tardías, los jóvenes españoles busquen en esta sustancia más una forma de conseguir estar activos durante muchas horas que una ayuda específica para mejorar el encuentro sexual.

Aunque nuestra muestra no es probabilística, resulta interesante apuntar las diferencias discursivas que se establecen respecto al alcohol y la cocaína en función del género del entrevistado. El alcohol, ya lo sabemos, es la droga más utilizada por todos, pero en especial las mujeres lo utilizarían más tanto para conseguir relaciones menos habituales, para aumentar la excitación y para prolongar las relaciones sexuales. La cocaína es valorada por ambos sexos y los hombres ven en la sustancia una forma de conseguir favores sexuales de las mujeres. El cannabis ya hemos visto que es poco valorado en general, pero algo más por el colectivo femenino.

Nos parece interesante destacar el papel que la gestión de la sexualidad cumple como mecanismo que potencia el uso de alcohol y otras drogas. Aunque es posible que exista algún mecanismo específico por el que las drogas afecten a la sexualidad, es también muy probable que su influencia sea indirecta. En este sentido, son importantes las expectativas y el contexto. Dado que cada vez más los contextos recreativos nocturnos de fin de semana son más utilizados por los jóvenes es necesario seguir investigando la relación entre uso de las drogas y la conducta sexual en estos contextos, así como los caminos a través de los que todo este entramado acaba por influir en las conductas de riesgo. Tanto el alcohol (Plant y Plant 2006; Abrahamson, 2004; Bellis y Hughes 2004; Boreham y McManus 2003; Ingham 2001), como el cannabis (Guo, Chung, Hill, Hawkins, Catalano y Abbot, 2002; Poulin et al, 2001, Brook, Balka y Whitemann, 1999), el éxtasis (McElrath, 2005) o la cocaína (Colfax, Vittinghoff, Husnik, McKirnan, Buchbinder, Koblin et al, 2004) están relacionadas con conductas sexuales de riesgo, aunque hemos de reconocer que la literatura no es unánime sobre este punto (Leigh, 2002). Cabe pensar, de cara a la prevención de conductas de riesgo, que esta relación tan positiva que algunos jóvenes han establecido entre sexualidad y drogas dificulta introducir elementos críticos que adviertan sobre los problemas asociados y la conveniencia de evitarlos.

Cabe pensar también que la prevención de las drogas debería también tener en cuenta como este tipo de sexualidad promovida desde los ambientes recreativos, rápida ("Fast sex") y estrictamente ligada a su función de dar placer, puede promover el consumo de drogas. ¿Existen posibilidades de promover otro tipo de sexualidad o es un camino sin retorno? Algunos autores (Otegui, 1998) verían una parte del problema en la adopción por parte de las mujeres de patrones de sexualidad atribuidos hasta ahora a los hombres.

\section{AGRADECIMIENTOS:}

A la Delegación del Gobierno para el Plan Nacional sobre Drogas que ha financiado a Socidrogalcohol parcialmente la investigación en 2006. A los colaboradores en el trabajo de campo en las tres CCAA: Agurtzane Castillo, Encarna Román, Nicole Blay, Ana López Durán, Elena Fernández del Río. 


\section{REFERENCIAS}

Abrahamson, M. (2004). Alcohol in courtship contexts: Focus-group interviews with young Swedish women and men. Contemporary Drug Problems, 31, 3-29.

Bantman, B. (1998). Breve historia del sexo. Barcelona. Paidos.

Baylen, C.A. y Rosenberg, H. (2006). A review of the acute subjective effects of MDMA/ecstasy. Addiction, 101, 933-947.

Beck J y Rosenbaum M (1994). Pursuit of Ecstasy: The MDMA Experience. Albany: State University of New York Press

Bellis, M.A. y Hughes K. (2004). Pociones sexuales Relación entre alcohol, drogas y sexo. Adicciones, 16, 251-60.

Bellis, M., Hughes, K., Dillon, P., Copeland, J. y Gates, P. (2007). Effects of backpacking holidays in Australia on alcohol, tobacco and drug use of UK residents. BMC Public Health, 7, 1-20.

Bellis, M. A., Hale, G., Bennet, L. A., Chaves, M. y Kilfoyle, M. (2000). Ibiza uncovered: changes in substance use and sexual behaviour amongst young people visiting an international night-life resort. Internacional Journal of Drug Policy, 11, 235-244.

Boreham, R. y McManus, S. (eds) (2003). Smoking, drinking and drug use among young people in England in 2002. Norwich: The Stationery Office.

Brook, D. W., Brook, J.S., Pahl, T., Montoya, I. (2002). The longitudinal relationship between drug use and risky sexual behaviors among Colombian adolescents. Archives of Pediatric Adolescente Medicine, 156, 1101-1107.

Brook, J. S., Balka, E. B. y Whiteman, M. (1999). The risks for late adolescence of early adolescent marijuana use. American Journal of Public Health, 89, 1549-1554.

Buffum, J., Moser, C. y Smith, D. (1988). Street drugs and sexual function. En J. M. A. Sitsen (Ed.), Handbook of Sexology. Volume VI. The pharmacology and endocrinology of sexual function. Amsterdam. Elsevier.

Calafat, A., Fernandez, C., Juan, M., Anttila, A., Bellis, M., Bohrn, K., Fenk, R., Hughes, K., Kerschl, A., Kokkevi, A., Kuussaari, K., Leenders, F., Mendes, F., Siamou, I., Simon, J., Wijngaart, G. y Zavatti, P. (2004) Cultural mediators in a hegemonic nightlife. Palma de Mallorca: Irefrea.

Calafat, A., Bohrn, K., Juan, M., Kokkevi, A., Maalsté, N., Mendes, F., Palmer, A., Sherlock, K., Simon, J., Stocco, P., Sureda, P., Tossmann, P., Wijngaart, G. y Zavatti, P (1999). Night life in Europe and recreative drug use. Sonar 98. Palma de Mallorca: IREFREA.

Calafat, A., Juan, M., Fernández, C., Gil, E. y Llopis, J.J. (2001) La vida social de la cocaína. Adicciones , 13 (supl 2), 61-103

Calafat, A., Juan, M., Becoña, E., Fernández, C., Gil, E., Palmer, A., Sureda, P., y Torres, M.A. (2000). Salir de marcha y consumo de drogas. Madrid: Plan Nacional sobre Drogas.

Cohen, S. (1982) Cannabis and sex: multifaceted paradoxes. Journal of Psychoactive Drugs, 14, 55-58.
Coleman, L. y Cater, S. (2005). Underage "binge" drinking: A qualitative study in to motivations and outcomes. Drugs: education, prevention and policy, 12, 125-136.

Colfax, G., Vittinghoff, E., Husnik, M. J.., McKirnan, D., Buchbinder, S., Koblin, B., Celum, C., Chesney, M., Huang, Y., Mayer, K., Bozeman, S., Judson, F. N., Bryant, K. y Coates, T. J. (2004). Substance use and sexual risk: A participant- and episode-level analysis among a cohort of men who have sex with men. American Journal of Epidemiology, 159, 1002-1012.

Collin, M. (1997). Altered State: The Story of Ecstasy Culture and Acid House. London: Serpent's Tail.

Comas, D (2005). Jóvenes y estilos de vida. Madrid. Fundación de Ayuda contra la Drogadicción.

Dawley, H. H., Winstead, D. K., Baxter, A. S. y Gay, J. R. (1979). An attitude survey of the effects of marijuana on sexual enjoyment. Journal of Clinical Psychology, 35, 212-217.

Dermen, K. H., Cooper, M. L. y Agocha, V. B. (1998) Sexrelated alcohol expectancies as moderators of the relationship between alcohol use and risky sex in adolescents. Journal of Studies on Alcohol, 59, 71-77.

Donovan, C. y McEwan, R. (1995) A review of the literature examining the relationship between alcohol use and HIV-related sexual risk-taking in young people. Addiction, 90, 319-328.

Elliott, L., Morrison, A., Ditton, J., Farral, S., Short, E., Cowan, L. y Gruer, L. (1998). Alcohol, drug use and sexual behaviour of young adults on a Mediterranean dance holiday. Addiction Research, 6, 319-340.

EMCDDA (2002). Luxemburgo: Recreational drug use - a key EU challenge. Drugs in focus 6. Office for Official Publications of the European Communities.

EMCDDA (2006) Annual report 2006: Selected issues. Recuperado el 3 de diciembre de 2007 de http:// issues06.emcdda.europa.eu/en/page024-en.html.

Espinosa, M. A. y Ochaita, E. (2003). Las practicas sexuales de los adolescentes y jóvenes españoles. Juventud y sexualidad 63. Recuperado el 3 de diciembre de 2007 de http://www.injuve.mtas.es/injuve/contenidos. downloadatt.action?id=2059896087.

Friedman, R. S., McCarthy, D. M., Förster, J. y Densier, M. (2005). Automatic effects of alcohol cues on sexual attraction. Addiction, 100, 672-681.

Gay, G. R., Newmeyer, J. A., Perry, M., Johnson G. y Kurland M. (1982). Love and haight: The sensuous hippy revisited. Drug/sex practices in San Francisco, 1980-81. Journal of Psychoactive Drugs, 14, 111-123.

Giddens, A. (1995). La transformación de la intimidad. Sexualidad, amor y erotismo en las sociedades modernas. Madrid: Gráficas Rógar.

Gilbert, J. y Pearson, E. (2003). Cultura y políticas de la música dance. Disco, hip-hop, house, techno, drum'n'bass y garage. Barcelona: Paidós.

Goodman, L. A. (1961). Snowball sampling. Annals of Mathematical Statistics, 32, 148-170.

Green, A. M. (1997). Des Jeunes et des Musiques. París: L'Harmattan. 
Green, A. I. y Halkitis, P. N. (2006). Crystal methamphetamine and sexual sociality in an urban gay subculture: an elective affinity. Cultural Health and Sex, 8, 317-333.

Guisado, J.A., Vaz, F.J., Fernández-Gil, M.A., Pacheco, D. Graell, M. y López-Ibor, J.J. (2000). Conducta sexual de riesgo en drogodependencias. Psiquiatría Biológica, 7, 91-94.

Guo, J., Chung, I. J., Hill, K. G., Hawkins, J. D., Catalano, R. F. y Abbott, R. D. (2002) Developmental relationships between adolescent substance use and risky sexual behavior in young adulthood. Journal of Adolescent Health, 3, 354-62.

Henderson, S. (1993). Fun, fashion \& frisson. International Journal of Drug Policy, 4, 122-129.

Hibell, B., Andersson, B., Bjarnasson, T., Ahlstrom, S., Balakireva, O., Kokkevi, A. et al. (2004). The 2003 ESPAD Report: Alcohol and Drug Use among Students in 30 European Countries. Stockholm: Swedish Council for information on Alcohol and other Drugs

INE (2004). Salud y hábitos sexuales. Las conductas sexuales desde la perspectiva del SIDA. Boletín Informativo del Instituto Nacional de Estadística 2004. Recuperado el 10 de marzo de 2005, de ttp://www.ine. es/revistas/cifraine/cifine_sida0704.pdf.

INJUVE (2004). Sondeos de opinión. Recuperado el 19 de marzo de 2005 de http://www.injuve.mtas.es/injuve/ contenidos .type .action? type $=292067219 \&$ menuld $=29$ 2067219

INJUVE (2005). La experiencia de la vida: aprendizaje y riesgos. La experiencia y las prácticas sexuales. Capitulo VI parte tercera. Recuperado el 5 de enero de 2006 de http://www.injuve.mtas.es/injuve/contenidos. item . action id $=2119765673 \&$ menuld $=549043401$

Ingham, R. (2001). Young people. Alcohol and sexual conduct. Sex Education Matters, 27, 9-10.

Kemmesles, U. (2000). How to reach the unknown: the snowball sampling technique. En G. Greenwod y K. Robertson (Eds.), Understanding and responding to drug use: The role of qualitative research (pp. 265-272). Luxembourg: European Monitoring Centre for Drugs and Drug Addiction (EMCDDA).

Leigh, B. C. (1990) The relationship of sex-related alcohol expectancies to alcohol consumption and sexual behavior. British Journal of Addiction, 85, 919-28

Leigh, B. C. (2002). Alcohol and condom use: a metaanalysis of event-level studies. Sexual and Transmission Diseases, 29, 476-82

López Blasco, A., Cachón, L., Comas, D., Andreu, J., Aguinaga, J. y Navarrete, L. (2005). Informe Juventud en España 2004. Madrid: MTAS, INJUVE.

Lyttle, T. y Montagne, M. (1992). Drugs, music and ideology: A social pharmacological interpretation of the acid house movement. International Journal of Addictions, 27, 1159-1177.

McElrath, K.(2005) MDMA and sexual behavior: ecstasy users' perceptions about sexuality and sexual risk. Substance Use and Misuse, 40, 1461-1477.

Megías, I. (2003). Jóvenes ante el sexo: Valores y expectativas asociadas. Revista de Estudios de Juventud, 63, 19-26.
Megias, I., Rodríguez, E., Mendez, S. y Pallares J. (2005). Jóvenes y sexo: el estereotipo que obliga y el rito que identifica. Madrid: Injuve y Fundación de Ayuda contra la Drogadicción (FAD).

Neale, J., Allen, D. y Coombes, L. (2005). Qualitative research methods within the addictions. Addiction, 100, 1584-1593.

Nicoll, A. y Hamers, F. F. (2002). Are trends in HIV, gonorrhoea, and syphilis worsening in western Europe? British Medical Journal, 324, 1324-1327.

Parsons, J. T., Kelly, B. C. y Wells, B. E. (2006). Differences in club drug use between heterosexual and lesbian/ bisexual females. Addictive Behaviors, 31, 2344-1349.

Plan Nacional sobre drogas (2004) Encuesta Estatal sobre el uso de drogas en Enseñanza Secundaria 2004. Recuperado el 3 de enero de 2005 de www.mir.es/pnd.

Plant, M. y Plant, M. (2006). Binge drinking. Alcohol and the national response. New York: Oxford University Press.

Poulin, C. y Graham, L. (2001). The association between substance use, unplanned sexual intercourse and other sexual behaviours among adolescent students. Addiction, 96, 607-621.

Rodríguez, J., Agulló, E. y Agulló, M. S. (2003). Jóvenes, fin de semana y uso recreativo de drogas: evolución y tendencias del ocio juvenil. Adicciones, 13 (supl 2), 7-33.

Rodríguez, A., Hernán, M., Cabrera, A., Romo, N., García, J.M. y Gutiérrez, J.L. (2006). ¿Tienen adolescentes y jóvenes que consumen drogas no inyectadas mayor probabilidad de transmisión sexual del VIH? Adicciones, 18, 61-72.

Russel, K. (1991). Lysergia suburbia, psychedelia: The acid house interpretation. En Unit for law and popular culture. Manchester: Polytechnic.

Sandelowski, M. (1995). Qualitative Analysis: What It Is and How to Begin, Research in Nursing \& Health, 18, 371-375.

Sumnall, H. R., Cole, J. C. y Jerome, L. (2006). The varieties of ecstatic experience: an exploration of the subjective experiences of ecstasy. Journal of Psychopharmacology, 20, 670-82

Sutherland, I. y Shepherd, J.P. (2002). Adolescents' beliefs about future substance use: a comparison of current users and non users of cigarettes, alcohol and illicit drugs. Journal of Adolescence, 25, 169-181.

Traeen, B., Hovland, A. y Odegard, G. (1998). Can I buy you a drink? Alcohol as symbolic communication in erotic encounters. Nordic Studies on Alcohol and Drugs, 15, 68-83.

UNAIDS (2006). Report on the global AIDS epidemic Recuperado el 5 de junio de 2006, de http://www. unaids.org/en/HIV_data/2006GlobalReport/default.asp.

Wilsnack, S. C. y Wilsnack, R. (2002). International gender and alcohol research: Recent findings and future directions. Alcohol Research and Health, 26, 245-250.

Zemishlany, Z., Aizenberg, D. y Weizman, A. (2001). Subjective effects of MDMA ('Ecstasy') on human sexual function. European Psychiatry, 16, 127-130. 
